\title{
Functional integration and abelian link invariants
}

\author{
Enore Guadagnini \\ October 20, 2018 \\ Dipartimento di Fisica, Università di Pisa \\ and INFN, Sezione di Pisa
}

\begin{abstract}
The functional integral computation of the various topological invariants, which are associated with the Chern-Simons field theory, is considered. The standard perturbative setting in quantum field theory is rewieved and new developments in the path-integral approach, based on the Deligne-Beilinson cohomology, are described in the case of the abelian $U(1)$ Chern-Simons field theory formulated in $S^{1} \times S^{2}$.
\end{abstract}

Talk given at the workshop Chern-Simons Gauge theory: 20 years after, Bonn, August 2009 


\section{Introduction}

The main subject of my talk concerns the use of the so-called path-integral —or functional integration - in the definition and in the computation of the various topological invariants which are associated with the quantum Chern-Simons field theory. This argument has already been introduced in several talks at this conference, so I shall skip the preliminaries and I will concentrate on the following question, which has indirectly been posed yesterday by one of the speakers.

We all agree that the path-integral has not a precise meaning. In particular, if $S_{C S}$ denotes the Chern-Simons action, the functional integral

$$
I(M)=\int_{M} D A e^{i S_{C S}[A]},
$$

which should correspond to an invariant of the 3-manifold $M$, is not well defined. So, what is the meaning of expression (1)?

I shall try to present the answer to this question in simple but rather complete terms. Some basic features of the use of the functional integration in quantum field theory will be described in section 2 and section 3. In section 4 some new developments in the path-integral computation of observables in Chern-Simons field theory will be presented. I will show how to bypass the difficulties of standard perturbation theory in the case of the abelian $U(1)$ ChernSimons theory formulated in a closed non-trivial 3-manifold $M$; the example $M=S^{1} \times S^{2}$ will be discussed in detail. A few observations on the 3-manifold invariants associated with the Chern-Simons theory are contained in section 5 and section 6 .

\section{Perturbative quantum field theory}

Functional integration can be used in perturbative quantum field theory. Given a set of fields - denoted by $\phi(x)$ - and an action functional $S[\phi]$, in physics one is usually interested in functional integrations with a quite peculiar "measure" which naively corresponds to the following product

$$
D \phi e^{i S[\phi]}=(\text { const. })\left(\prod_{x} d \phi(x)\right) e^{i S[\phi]} .
$$

Alternatively, one can introduce a complete set of orthonormal functions $\left\{\psi_{n}(x)\right\}$, with $n \in \mathbb{N}$, so that each classical configuration $\phi(x)$ can be written as a linear combination of the base functions, $\phi(x)=\sum_{n} c_{n} \psi_{n}(x)$. By varying the (real) coefficients $\left\{c_{n}\right\}$ one gets an infinitesimal variation $d \phi(x)$ of the fields, $d \phi(x)=\sum_{n} d c_{n} \psi_{n}(x)$. Then, one can replace expression (2) with

$$
D \phi e^{i S[\phi]}=\text { (const.) }\left(\prod_{n=1}^{\infty} d c_{n}\right) e^{i S[\phi]} .
$$

It is well known that both expressions (2) and (3) are not well defined. With an infinite number of integration variables, the result of an integration looks like the product of an 
infinite number of real coefficients that, apart from very exceptional cases, is not well defined. So, independently of the choice of the value of the multiplicative constant which appears in equation (2) or in equation (3), both expressions (2) and (3) do not represent a well defined integration measure. In facts, the integral of the measure $I=\int D \phi e^{i S}$, which can be imagined to represent some kind of "partition function" — precisely like expression (1) — is in general not well defined.

\subsection{Observables}

On the other hand, it is a fact that perturbative quantum field theory provides a rather accurate description of physical phenomena. For instance, in the $S U(3)_{c} \times S U(2)_{L} \times U(1)_{Y}$ Standard Model, by means of the functional integration method one can compute the value of the magnetic moment of the electron (or of the other charged leptons). The prediction of the Standard Model can be compared with the observed experimental value of the magnetic moment of the electron. The actual agreement [1] between the computed value and the real observed value is within less then one part in a million. In addition to the remarkable correspondence of the experiments with the predictions of quantum field theory, the puzzling question is:

How is it possible, by using a ill-defined functional integration, to make a prediction?

For, in order to make a prediction, no ambiguities must occur, all the steps of the computation have to be well defined and, independently of any choice of notations or conventions, the final expression/value of the prediction must be unique.

The point is that all the predictions of quantum field theory that can be compared with experiments - quantities of this type are called "observables" - never take the form of the partition function $I=\int D \phi e^{i S}$. In standard quantum field theory, the observables are strictly connected with expectation values of the type

$$
\langle F[\phi]\rangle=\frac{\int D \phi e^{i S[\phi]} F[\phi]}{\int D \phi e^{i S[\phi]}}
$$

where $F[\phi]$ is a functional of the fields. At first sight, expression (4) also appears to be not well defined because, in order to compute $\langle F[\phi]\rangle$, one could imagine to evaluate separately the numerator and the denominator and then to take the ratio. However, this is not what physicists do in order to compute the expectation values. In standard perturbative quantum field theory equation (4) means: choose some kind of "regularization" in order to give a meaning to the numerator and to the denominator simultaneoulsy. Then, for the regularized ratio, consider the limit in which the regularization is removed. This limit exists, or it exists at least for the quantum field theory models that appear to be somehow related to the description of physical phenomena. I shall present one example in a while. The regularization of the path-integral measure must necessarily be expressed in terms of a finite number of integration variables. Thus, for example, one could regularize expression (3) by considering 
the finite product

$$
\left.D \phi e^{i S[\phi]}\right|_{\mathrm{reg}}=D \phi_{N} e^{i S[\phi]}=\text { (const.) }\left(\prod_{n=1}^{N} d c_{n}\right) e^{i S[\phi]} .
$$

In this case, expression (4) should be interpreted as

$$
\langle F[\phi]\rangle=\lim _{N \rightarrow \infty} \frac{\int D \phi_{N} e^{i S[\phi]} F[\phi]}{\int D \phi_{N} e^{i S[\phi]}} .
$$

After this first logical settlement, the next step consists in disentangling the difficulties in the computation of $\langle F[\phi]\rangle$ that are related to the form of the action $S[\phi]$ from those that are connected with the structure of the functional $F[\phi]$ itself. To this end, it is convenient to consider the so-called correlation functions

$$
\left\langle\phi\left(x_{1}\right) \cdots \phi\left(x_{n}\right)\right\rangle=\frac{\int D \phi e^{i S[\phi]} \phi\left(x_{1}\right) \cdots \phi\left(x_{n}\right)}{\int D \phi e^{i S[\phi]}} .
$$

(From now on it is understood that the meaning of a ratio of two functional integrals, like expression (7), is specified by a prescription of the type illustrated in equation (6).) The correlation functions are determined by the form of the action $S[\phi]$ and, provided they are well defined, one can then consider the problem of expressing $\langle F[\phi]\rangle$ in terms of the correlation functions. In several field theory applications in particle physics, this second task is rather trivial because from the correlation functions one can obtain directly the transition amplitudes for the various particles processes. However, in the Chern-Simons field theory, the solution of this second problem presents peculiar non-trivial aspects. In the computation of the expectation values of the holonomies associated with oriented links, one has to introduce a framing procedure in order to eliminate the ambiguities - which occur in the product of two $A$ fields at the same point- and preserve the ambient isotopy invariance.

In order to control the whole set of correlation functions, it is useful to introduce the generating functional

$$
Z[J]=\frac{\int D \phi e^{i S[\phi]} e^{i \int J \phi}}{\int D \phi e^{i S[\phi]}}
$$

where $J=J(x)$ is a classical "source" variable. Perturbative quantum field theory is based on the existence of the so-called "free" fields. Hence, let us illustrate the meaning of expression (8) in the simple case in which the action is a quadratic functional $S=S_{0}$ of the fields

$$
S_{0}[\phi]=\frac{1}{2} \int d x \phi(x) \nabla \phi(x),
$$

where $\nabla$ is an appropriate differential operator 1 . The following identity, which is not related to the path-integral at all, plays an important role. One has

$$
\begin{aligned}
e^{i S_{0}[\phi]} e^{i \int J \phi} & =\exp \left\{\frac{i}{2} \int d x[\phi(x) \nabla \phi(x)+2 J(x) \phi(x)]\right\} \\
& =\exp \left\{\frac{i}{2} \int d x \widetilde{\phi}(x) \nabla \widetilde{\phi}(x)\right\} \exp \left\{-\frac{i}{2} \int d x d y J(x) \nabla^{-1}(x, y) J(y)\right\} \\
& =e^{i S_{0}[\widetilde{\phi}]} e^{-\frac{i}{2} \int J \nabla^{-1} J},
\end{aligned}
$$

\footnotetext{
${ }^{1}$ For instance, when the field model is used to describe one kind of free spinless particles, the operator $\nabla$ is given by $\nabla=-\eta^{\mu \nu} \partial^{2} / \partial x^{\mu} \partial x^{\nu}-m^{2}$, where $\eta^{\mu \nu}$ denotes the Minkowski metric.
} 
where

$$
\widetilde{\phi}(x)=\phi(x)+\int d y \nabla^{-1}(x, y) J(y) \equiv \phi(x)+\text { const. },
$$

and $\nabla^{-1}(x, y)$ is a Green function for the $\nabla$ operator

$$
\nabla \cdot \nabla^{-1}(x, y)=\delta(x-y)
$$

In general, $\nabla^{-1}(x, y)$ satisfies certain analytic propertie 2 which, in particle physics, must be consistent with some observed properties of the experimental data like causality and energy positivity. For the moment, let us assume that $\nabla^{-1}(x, y)$ exists, I shall return to this point later. The identity (10) implies that the generating functional $Z_{0}[J]$ for free fields can be written as

$$
Z_{0}[J]=e^{-\frac{i}{2} \int J \nabla^{-1} J} \times \frac{\int D \phi e^{i S_{0}[\phi+\text { const. }]}}{\int D \phi e^{i S_{0}[\phi]}} .
$$

Now one finds the crucial point. In order to compute the ratio of the two functional integrations shown in equation (13), one must consider the limit (in which the regularization is removed) of regularized functional integrations. With a finite number of integration variables, the result of the integral is invariant under translation of these variables, thus

$$
\frac{\int D \phi e^{i S_{0}[\phi+\text { const. }]}}{\int D \phi e^{i S_{0}[\phi]}} \equiv \lim _{N \rightarrow \infty} \frac{\int D \phi_{N} e^{i S_{0}[\phi+\text { const. }]}}{\int D \phi_{N} e^{i S_{0}[\phi]}}=1,
$$

and the generating functional is then

$$
Z_{0}[J]=\exp \left\{-\frac{i}{2} \int d x d y J(x) \nabla^{-1}(x, y) J(y)\right\} .
$$

Note that, even if the computation of $Z_{0}[J]$ that has been presented here is somehow based on the functional integration method, in the whole argument no ill-defined functional integration has been really computed. $Z_{0}[J]$ is well defined and determines the value of all the observables of the free theory. For instance, the poles in the Fourier transform of $\nabla^{-1}(x, y)$ fix the value of the particles mass. So, all the observables of the free theory do not depend at all on the value that one could imagine to give to the partition function $I_{0}=\int D \phi e^{i S_{0}}$. This remains true also in the case of interacting fields models, where the action contains cubic or quartic terms in powers of the fields. In fact, perturbative quantum field theories can also be formulated 2, 3, without the introduction of functional integration.

Remark. Equation (14) can also be interpreted as a defining relation, because equality (14) is precisely the only property of the functional integration that is used in standard perturbative quantum field theory.

To sum up, in the path-integral formulation of perturbative quantum field theories there is really no need of computing the partition function $I=\int D \phi e^{i S}$, any functional integration

\footnotetext{
${ }^{2}$ The Green function $i \nabla^{-1}(x, y)$ is usually called the Feynman propagator and, in the case of free spinless particles, it is given by $i \nabla^{-1}(x, y)=i \int\left[d^{4} p /(2 \pi)^{4}\right] e^{-i p(x-y)}\left(p^{2}-m^{2}+i \epsilon\right)^{-1}$.
} 
of this type is not well defined 3 and all the observables do not depend on it. Clearly, the fact that the functional integration $I=\int D \phi e^{i S}$ is not well defined is not connected with the possible existence of an analytic continuation of the field model in the euclidean region (this subject is related to the analytic properties of the Feynman propagator). Also, as far as the observables of quantum field theory are concerned, the question whether, in the functional integration, one has to sum over smooth or singular configurations for the field variables is a completely irrelevant issue.

Finally, in analogy with the result of a gaussian integral with a finite number of integration variables, sometimes in literature one finds the relation

$$
I_{0}=\int D \phi e^{i S_{0}}=(\text { const. }) \operatorname{Det}^{-1 / 2}(-i \nabla) .
$$

Equation (16) is not a definition of the value of the partition function because the expression appearing on the r.h.s. (for the differential operators $\nabla$ that normally enter the action functional) is not well defined. Expression (16) can be used as a guess-suggesting reminder for the properties of the regularized functional integral. For instance, when $\nabla$ smoothly depends on a parameter (or on a set of parameters) $\lambda$, from equation (16) one can guess the expression for the logarithmic variation of $I_{0}$ with respect to $\lambda$,

$$
I_{0}^{-1} \frac{\partial I_{0}}{\partial \lambda}=-\frac{1}{2} \operatorname{Tr}\left(\nabla^{-1} \frac{\partial \nabla}{\partial \lambda}\right)
$$

Differently from equation (16), expression (17) is well defined and, in facts, its structure is similar to the structure of the correlation functions - or of many of the observables - in quantum field theory [4].

In a "free fields" model, the correlations functions can be derived from expression (15),

$$
\left\langle\phi\left(x_{1}\right) \cdots \phi\left(x_{n}\right)\right\rangle_{0}=\frac{\int D \phi e^{i S_{0}[\phi]} \phi\left(x_{1}\right) \cdots \phi\left(x_{n}\right)}{\int D \phi e^{i S_{0}[\phi]}}=\left.\frac{(-i)^{n} \delta^{n} Z_{0}[J]}{\delta J\left(x_{1}\right) \cdots \delta J\left(x_{n}\right)}\right|_{J=0} .
$$

If the functional $F[\phi]$ can be written as a smooth linear combination of the correlation functions, one can then evaluate the observable $\langle F[\phi]\rangle$. Since the correlations functions are really distributions, the computation of $\langle F[\phi]\rangle$ may present ambiguities when, for instance, a correlation function is integrated with a function that is not a test function or when, in a correlation function, one needs to consider the limit of two (or more) coincident points. This problem, which is also present in an interacting fields model, is related (in part) to the so-called composite operators problem.

\footnotetext{
${ }^{3}$ Something similar also happens in statistical mechanics where the partition function $Z$, which takes the form $Z=\operatorname{Tr} e^{-H / k T}$, is not an observable. The observables are combinations of the normalized mean values or can be derived from the thermodynamic potentials in the thermodynamic limit. For example, in order to determine the free energy of the system, one only needs to consider the leading term of the expansion of $\ln Z$ in powers of the volume (for instance) in the thermodynamic limit. As a result, if one modifies the partition function and multiplies it by, say, a factor five, $Z \rightarrow Z^{\prime}=5 Z$, the expression of the free energy does not change. Thus, $Z$ cannot be - and in facts it is not - an observable, whereas the free energy is.
} 


\subsection{Interactions and renormalization}

In the case of an interacting fields model, the action $S[\phi]$ is written as the sum of two terms, $S[\phi]=S_{0}[\phi]+S_{I}[\phi]$, where $S_{0}$ denotes the "free" action and $S_{I}$ contains the interaction terms. The generating functional $Z[J]$ of equation (8) can be written as

$$
Z[J]=e^{i Z^{c}[J]}=\frac{\int D \phi e^{i S_{0}} e^{i S_{I}} e^{i \int J \phi}}{\int D \phi e^{i S_{0}}} / \frac{\int D \phi e^{i S_{0}} e^{i S_{I}}}{\int D \phi e^{i S_{0}}}
$$

where the numerator

$$
\left\langle e^{i S_{I}} e^{i \int J \phi}\right\rangle_{0}=\frac{\int D \phi e^{i S_{0}} e^{i S_{I}} e^{i \int J \phi}}{\int D \phi e^{i S_{0}}}
$$

denotes the sum of all the Feynman diagrams (which are constructed with the Feynman propagator $i \nabla^{-1}$, the interaction vertices of $S_{I}$, and in which each external leg corresponds to $\left.-\int d y \nabla^{-1}(x, y) J(y)\right)$; and the denominator

$$
\left\langle e^{i S_{I}}\right\rangle_{0}=\frac{\int D \phi e^{i S_{0}} e^{i S_{I}}}{\int D \phi e^{i S_{0}}}
$$

just corresponds to the sum of the vacuum-to-vacuum diagrams, i.e. diagrams with no external legs. Actually, the sum of the vacuum-to-vacuum diagrams factorizes in the numerator and cancels out with the denominator. So, there is no need of computing the vacuum-to-vacuum diagrams (which remain divergent even after the standard regularization/renormalization procedure has been introduced). In conclusion, in the derivation of the correlation functions and of the observables of an interacting field theory, one never has to compute the value of the partition function $I=\int D \phi e^{i S}$.

A few remarks on the meaning of the renormalization in field theory are in order. By means of a Legendre transformation of the functional $Z^{c}[J]$ of the connected correlation functions, one can introduce the effective action $\Gamma[\varphi]$,

$$
\varphi(x)=\frac{\delta Z^{c}[J]}{\delta J(x)} \quad, \quad \Gamma[\varphi]=Z^{c}[J]-\int d x J(x) \varphi(x),
$$

which is the sum of the one-particle-irreducible Feynman diagrams in which the external legs are represented by the classical variable $\varphi$. The perturbative expansion of a generic (nontrivial) correlation function — of the field theory defined by the action $S[\phi]$ - is equal to the perturbative expansion containing diagrams at the tree level only of a new field model defined by the action that coincides with the functional $\Gamma[\varphi]$. Diagrams at the tree level contain no loops: so, they present no ultraviolet divergences and maintain all the symmetries of the action. This means that, provided $\Gamma[\varphi]$ is well defined, all the correlations functions are well defined. Since the effective action establishes how the symmetries of the theory are realized and determines the values of the observables $4, \Gamma[\varphi]$ is the fundamental functional that must be considered in the renormalization task.

\footnotetext{
${ }^{4}$ The magnetic moment of the electron, for instance, is described by the three-point proper vertex containing two spinor electron fields and one vector electromagnetic field. Namely, the renormalized effective action $\Gamma$ of the Standard Model admits an expansion in powers of the fields. Consider now the term $B \in \Gamma$ given
} 
Quite often, some of the diagrams contributing to $\Gamma$ are not well defined and present ambiguities. The root of these ambiguities is usually due to the presence of divergences which can be eliminated (in agreement with the action principle) by local conterterms, i.e. by terms which have the form of integrals of polynomials of the field variables (and their derivatives) defined in the same point with divergent coefficients. Generally, in the intermediate steps of the renormalization, one makes use of an arbitrary regularization and, after the introduction of appropriate local counterterms (which also depend on the choice of the regularization), one takes the limit in which the regularization is removed. The whole renormalization procedure consists of:

- introduction of local counterterms (with divergent and finite coefficients) in the diagrams computations in order to make the effective action $\Gamma$ finite and maintain the maximum number of symmetries (Lorentz symmetry, internal and gauge symmetries,...);

- introduction of the normalization conditions, which determine the meaning of the finite physical parameters on which the renormalized $\Gamma$ eventually depends.

In renormalizable models, only a finite number of parameters need to be fixed (coupling constants, particle masses, fields or wave functions normalizations). The finite values of the coupling constants and of the particle masses - that must agree with the experimental values - are also called the renormalized or physical parameters. The coupling constants usually correspond to the values of certain transition amplitudes in particular kinematic conditions (for instance, in the limit of vanishing momenta), and the particle masses corresponds to the poles in the energy variable of the dressed propagators. This means that, in the case of an interacting field theory, a few specific terms of $\Gamma$ (and not of the action $S$ ) determine the values of the renormalized or physical parameters. The renormalized parameters are observables, whereas the so-called bare parameters — which enter the action functional $S[\phi]$ are not observables.

The abelian Chern-Simons field theory in $\mathbb{R}^{3}$ is a "free fields" theory because the action is a quadratic functional of the fields $S_{C S}=2 \pi k \int A \wedge d A$; the renormalization is trivial in this case because the effective action coincides with the action.

The Chern-Simons field theory in $\mathbb{R}^{3}$ with a simple non-abelian gauge group is an interacting theory and a non-trivial renormalization is required because some of the contributions to the effective action have ambiguities. For instance, the one-loop correction to the two point function for the connection field $A$ is the sum of two terms: each term is divergent but, in their sum, the two divergent parts tend to cancel. Consequently, this sum is not well defined $(\infty-\infty$ is not well defined) and has ambiguities. In agreement with the behaviour of all perturbative quantum field theories, these ambiguities take the form of a finite local term, namely $b \int A^{a} \wedge d A^{a}$, where $b$ is an arbitrary finite parameter. Since this term has

by $B=\int d x d y d z \bar{\psi}_{\alpha}(x) \Lambda_{\alpha \beta}^{\mu}(x, y, z) \psi_{\beta}(y) A_{\mu}(z)$, where $\psi(x)$ is the spinor field associated with the electron and $A_{\mu}(x)$ denotes the 4 -vector potential of electromagnetism. The function $\Lambda_{\alpha \beta}^{\mu}(x, y, z)$ (three-point proper vertex) describes how the electron interacts with the electromagnetic field and contains, in particular, the required information on the magnetic moment of the electron. 
the same structure of a lagrangian term, the ambiguity in the value of $b$ is totally irrelevant and produces no physical observable effects. (This kind of ambiguities, which is well known in quantum field theory, concerns the finite terms arbitrariness in the renormalization process.) In facts, any choice of the finite value of $b$ does not modify the structure of the proper vertices contained in $\Gamma$ (it simply changes the name of some bare unobservable parameter); consequently, the perturbative expansion of a generic correlation function - which is equal to the perturbative expansion made of tree-level diagrams only, constructed with the functional $\Gamma$ - is not modified by a change of the value of $b$. Thus, in the non-abelian Chern-Simons field theory in $\mathbb{R}^{3}$ with compact gauge group, the non-trivial aspect of the renormalization is concentrated in the normalization conditions, which state how to identify the coupling constant. In particular, consider the complete (i.e. the sum of the contributions to all orders of perturbation theory) two-point proper vertex for the $A$ field contained in $\Gamma$; its structure is fixed by the symmetries of the theory and takes the form

$$
\left.\Gamma\right|_{A A}=\alpha \int A^{a} \wedge d A^{a}
$$

where the nonvanishing real coefficient $\alpha$ admits a power expansion in terms of the bare parameters. The dependence of $\alpha$ on the bare parameters is not unique and can be arbitrarily modified by changing the regularization; but how $\alpha$ depends on the bare parameters is not observable and then it is totally irrelevant. When the renormalized $\Gamma$ preserves the BRS symmetry, the normalization condition states that the coupling constant $k$ of the ChernSimons theory is given by

$$
\alpha=k / 8 \pi \text {. }
$$

Like in any renormalizable field theory, all observables depend unambiguosly 5 on the renormalized parameters (in our case, on $k$ ). In particular, a second order computation in perturbation theory of the expectation values of the holonomies shows that, in terms of the coupling constant $k$, the deformation parameter $q$ turns out to be

$$
q=e^{-2 \pi i / k}
$$

The coupling constant of the non-abelian Chern-Simons field theory in three dimensions should not be confused with the so-called "level" parameter which appears in two-dimensional conformal field theories.

\section{Perturbative Chern-Simons field theory}

Let us now concentrate on the Chern-Simons field theory [5] and on the path-integral computation of the topological invariants. It is important to distinguish the cases in which the topological model is defined in $\mathbb{R}^{3}$ or in a closed 3-manifold $M$. Also, it is significant to

\footnotetext{
${ }^{5}$ For example, in the Standard Model the magnetic moment of the electron depends unambiguosly [1] on the value of the renormalized electromagnetic coupling constant $\alpha_{e m} \simeq 1 / 137$. Whereas the dependence of $\alpha_{e m}$ on the "bare" coupling constant is not unique and is not observable; in facts, can you imagine how to measure it by means of an experiment?
} 
distinguish the expectation values $\langle W[A]\rangle$, of a gauge invariant functional $W[A]$ of the $A$ fields, from (a possible variant of) the partition function $I=\int D A e^{i S_{C S}}$.

Let us firstly recall the results obtained in perturbation theory in $\mathbb{R}^{3}$. The non-abelian Chern-Simons field theory formulated in $\mathbb{R}^{3}$ with compact gauge group is perturbatively renormalizable. Several features of the perturbative expansion have been explored with the covariant gauge-fixing of the so-called Landau gauge (explicit computations have been produced up to two loops). The primitive ultraviolet divergences associated with the single diagrams - constructed with the connections and ghosts fields - tend to cancel in the construction of the proper vertices. Actually, the theory is finite [6] to all orders of perturbation theory. Therefore, in the renormalization process, only the finite local conterterms freedom remains to be fixed by means of the normalization conditions. These conditions determine how to identify the coupling constant in the effective action.

Since renormalizability is determined by the short-distance behaviour of the model, the ultraviolet properties of the Chern-Simons theory formulated in $\mathbb{R}^{3}$ or formulated in a generic 3 -manifold $M$ are obviously the same. This does not imply that the use of perturbation theory extends trivially from $\mathbb{R}^{3}$ to $M$. There are in fact obstacles — for both the abelian and the non-abelian Chern-Simons theories formulated in a closed 3-manifold $M$ - to produce real perturbative computations of the observables, but these difficulties are not related to the ultraviolet divergences.

For the abelian Chern-Simons theory in $\mathbb{R}^{3}$, the expectation values of the holonomies, which are associated with coloured oriented and framed links, can explicitly be computed in closed form by means of standard perturbation theory.

In the non-abelian case with compact gauge group, the explicit computation of the expectation values of the Wilson line operators in $\mathbb{R}^{3}$ has been produced at the third non-trivial order of perturbation theory [7]. The results of perturbation theory are in agreement with what is expected on the basis of general arguments. In facts, by taking into account the relevant symmetry properties of the expectation values in quantum field theory - namely, ambient isotopy invariance, validity of satellite relations, structure of the representation ring of the gauge group, covariance of the expectation values under a modification of the framingone finds that the expression of the expectation values (of the trace of the holonomies) is unique. These invariants of framed and coloured links take the form of generalized Jones polynomials [8]; the algebraic structure of these polynomials, which is determined by the characters of simple Lie groups, is very general. In facts, these link invariants can also be obtained or defined by means of skein relations [9], quantum group Hopf algebra methods [10, statistical state models [11. For each simple Lie algebra, the corresponding braid group representations entering the construction of these link polynomials have a universal and canonical structure (the classification of these braid group representations is somewhat similar to the classification of the irreducible representations of simple Lie algebras). These braid group representations also appear, for example, as monodromy representations in conformal bidimensional models [12, 13, 14].

The perturbative setting of the Chern-Simons field theory can be imagined to be extended 
from $\mathbb{R}^{3}$ to a generic closed 3-manifold $M$. Indeed, provided the fields propagator is well defined, the whole perturbative construction based on the Wick contractions trivially follows. However, as a matter of facts, no explicit example of a real functional integral computation -in standard perturbation theory - of an observable in a closed 3-manifold $M \neq S^{3}$ has been produced in the last twenty years.

\section{Functional integral and Deligne-Beilinson cohomology}

When the Chern-Simons field theory is formulated in a nontrivial closed oriented manifold $M \neq S^{3}$, the explicit computation of the observables by means of the standard perturbation theory within the path-integral method presents technical difficulties, which are related to the gauge-fixing procedure and the definition of the fields propagator. Let me show how it is possible to overcome these problems in the abelian case [15].

\subsection{Basic definitions}

The abelian Chern-Simons theory [16, 17, 18, with gauge group $U(1)$ is defined by means of a $U(1)$-connection $\mathrm{A}$ in a closed oriented 3-manifold $M$. The holonomy associated with an oriented knot $C \subset M$ is given by the integral $\int_{C} A$; this integral is invariant under ordinary $U(1)$ gauge transformations acting on $A$. In the standard field theory formulation of abelian gauge theories, the configuration space locally coincides with the set of 1-forms modulo exact forms, $A \sim A+d \Lambda$. But if one assumes [5, 19] that a complete set of observables is given by the exponential of the holonomies $\left\{\exp \left[2 \pi i \int_{C} A\right]\right\}$ which are associated with oriented knots $C$ in $M$, the invariance group of the observables is actually larger than the standard gauge group; in facts, the observables are locally defined on the classes of 1-forms modulo forms $\widehat{A}$ with integer periods, $A \sim A+\widehat{A}, \int_{C} \widehat{A}=n \in \mathbb{Z}$. More precisely, the configuration space is defined in terms of the Deligne-Beilinson (DB) cohomology classes 20, 21, 19.

The DB class associated with the connection $A$ will be denoted by the same symbol $A \in H_{D}^{1}(M)$, where $H_{D}^{1}(M)$ represents the DB cohomology group of $M$ of degree 1 . Let $H_{D}^{3}(M)$ be the space of the DB classes of degree 3; the *-product of two classes is a pairing of the DB cohomology groups that defines a natural mapping 22$]$

$$
H_{D}^{1}(M) \otimes H_{D}^{1}(M) \longrightarrow H_{D}^{3}(M) ;
$$

the *-product of $A$ with $A$ just corresponds to the abelian Chern-Simons lagrangian

$$
A * A \longrightarrow A \wedge d A \text {. }
$$

Like the integral of any element of $H_{D}^{3}(M)$, the Chern-Simons action

$$
S_{C S}[A]=\int_{M} A * A \longrightarrow \int_{M} A \wedge d A
$$

is defined modulo integers; consequently, the path-integral phase factor

$$
e^{2 \pi i k S_{C S}[A]}=e^{2 \pi i k \int_{M} A * A}
$$


is well defined when the coupling constant $k$ takes integer values, $k \in \mathbb{Z},(k \neq 0)$. Let us now consider a framed, oriented and coloured link $L \subset M$ with $N$ components $\left\{C_{1}, C_{2}, \ldots, C_{N}\right\}$. The colour of each component $C_{j}$, with $j=1,2, \ldots, N$, is represented by an integer charge $q_{j} \in \mathbb{Z}$. The classical expression $W(L)$ of the Wilson line is given by

$$
W(L)=\prod_{j=1}^{N} \exp \left\{2 \pi i q_{j} \int_{C_{j}} A\right\}=\exp \left\{2 \pi i \sum_{j} q_{j} \int_{C_{j}} A\right\},
$$

and the observables of the Chern-Simons gauge theory in $M$ are given by the expectation values

$$
\left.\langle W(L)\rangle\right|_{M}=\frac{\int_{M} D A e^{2 \pi i k S_{C S}[A]} W(L)}{\int_{M} D A e^{2 \pi i k S_{C S}[A]}},
$$

where the path integral should be defined on the DB classes which belong to $H_{D}^{1}(M)$.

Note. One usually assumes that expression (28) is well defined. But one should keep in mind that, for certain manifolds $M$ and for certain values of the coupling constant $k$, expression (28) could not be well defined.

The structure of the functional space admits a natural description in terms of the homology groups of $M$, as indicated by the following exact sequence [23, 24]

$$
0 \longrightarrow \Omega^{1}(M) / \Omega_{\mathbb{Z}}^{1}(M) \longrightarrow H_{D}^{1}(M) \longrightarrow H^{2}(M) \longrightarrow 0,
$$

where $\Omega^{1}(M)$ is the space of 1-forms on $M, \Omega_{\mathbb{Z}}^{1}(M)$ is the space of closed 1-forms with integer periods on $M$ and $H^{p}(M)$ is the $(p)^{t h}$ integral cohomology group of $M$. Thus, $H_{D}^{1}(M)$ can be understood as an affine bundle over $H^{2}(M)$, whose fibres have a typical underlying (infinite dimensional) vector space structure given by $\Omega^{1}(M) / \Omega_{\mathbb{Z}}^{1}(M)$.

\subsection{Distributional forms}

Now, the crucial observation is that, in order to compute the observables (28), the introduction of a gauge-fixing and of the fields propagator is not essential. In order to illustrate this point, let us consider first the case $M=S^{3}$. The integral of a one-form $A$ along an oriented knot $C \subset S^{3}$ can be written as the integral on the whole $S^{3}$ of the external product $A \wedge J_{C}$, where the current $J_{C}$ is a distributional 2-form with support on the knot $C$; that is, one can write $\int_{C} A=\int_{S^{3}} A \wedge J_{C}$. Since $C$ can be understood as the boundary of a Seifert surface $\Sigma_{C}$ in $S^{3}$, one has $J_{C}=d \eta_{C}$ for some 1-form $\eta_{C}$ with support on $\Sigma_{C}$. One then finds $\int_{C} A=\int_{S^{3}} A \wedge d \eta_{C}$. For example, consider the unknot $C$ in $S^{3}$ shown in Figure 1, with a simple disc as Seifert surface. Inside the open domain depicted in Figure 1, the oriented knot is described - in local coordinates $(x, y, z)$ - by a piece of the $y$-axis and the corresponding distributional forms $J_{C}$ and $\eta_{C}$ are given by

$$
J_{C}=\delta(z) \delta(x) d z \wedge d x \quad, \quad \eta_{C}=\delta(z) \theta(-x) d z .
$$

In terms of DB classes, one has

$$
\exp \left\{2 \pi i \int_{C} A\right\}=\exp \left\{2 \pi i \int_{S^{3}} A * \eta_{C}\right\},
$$


where $\eta_{C}$ denotes the DB class - associated with the knot $C$ - that is locally represented by the distributional form $\eta_{C}$.

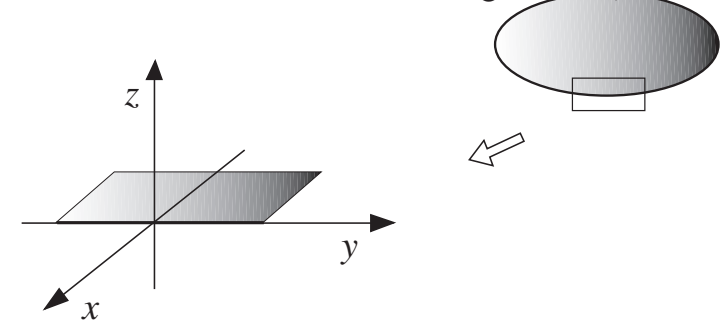

Figure 1: In a open domain with local coordinates $(x, y, z)$, a piece of the knot $C$ can be identified with the $y$ axis, and the disc that it bounds can be identified with a portion of the half plane $(x<0, y, z=0)$.

For the coloured link $L \subset S^{3}$, one can write

$$
W(L)=\exp \left\{2 \pi i \sum_{j} q_{j} \int_{C_{j}} A\right\}=\exp \left\{2 \pi i \int_{S^{3}} A * \eta_{L}\right\}
$$

where $\eta_{L}=\sum_{j} q_{j} \eta_{j}$ denotes the DB class associated with the link $L$. Since $H^{2}\left(S^{3}\right)$ is trivial, sequence (29) implies $H_{D}^{1}\left(S^{3}\right) \simeq \Omega^{1}\left(S^{3}\right) / \Omega_{\mathbb{Z}}^{1}\left(S^{3}\right)$. The analogue of equation (10) now takes the form

$$
\begin{aligned}
e^{2 \pi i k S_{C S}[A]} W(L) & =\exp \left\{2 \pi i k \int_{S^{3}} A * A+2 \pi i \int_{S^{3}} A * \eta_{L}\right\} \\
& =\exp \left\{2 \pi i k \int_{S^{3}} \widetilde{A} * \widetilde{A}\right\} \exp \left\{-(2 \pi i / 4 k) \int_{S^{3}} \eta_{L} * \eta_{L}\right\} \\
& =e^{2 \pi i k S_{C S}[\widetilde{A}]} \exp \left\{-(2 \pi i / 4 k) \int_{S^{3}} \eta_{L} * \eta_{L}\right\},
\end{aligned}
$$

where $\widetilde{A}=A-(1 / 2 k) \eta_{L}$. The ambiguities in $\int_{S^{3}} \eta_{L} * \eta_{L}$ that are related to the self-linking number can be fixed in the standard way by the introduction of a framing for the link $L$. At this point, assuming invariance under translation of the functional integral (as shown in equation (14)), one finally gets

$$
\left.\langle W(L)\rangle\right|_{S^{3}}=\exp \left\{-(2 \pi i / 4 k) \int_{S^{3}} \eta_{L} * \eta_{L}\right\}=\exp \left\{-(2 i \pi / 4 k) \sum_{i j} q_{i} \mathbb{L}_{i j} q_{j}\right\},
$$

where $\mathbb{L}_{i j}$ are the matrix elements of the linking matrix associated with the link $L$. Equation (33) coincides with the result of standard perturbation theory but, in the derivation of expression (33), both gauge-fixing and Feynman propagator have not been introduced.

The DB formalism turns out to be particularly useful for the path-integral computation of the observables in a generic 3-manifold $M$ because sequence (29) describes the non-trivial structure of the functional space and equation (30) remains valid also in the case of a $\operatorname{knot} C$ in a generic 3-manifold $M$. Indeed, the class $\eta_{C} \in H_{D}^{1}(M)$ which is canonically associated with a knot $C \subset M$ is well defined for arbitrary 3-manifold $M$. 


\subsection{Observables in non-trivial manifolds}

As an example of functional integration in a non-trivial manifold, let us consider the DeligneBeilinson formalism when the Chern-Simons theory is formulated in the manifold $M=$ $S^{1} \times S^{2}$, which can be represented by the region of $\mathbb{R}^{3}$ which is delimited by two concentric 2 -spheres, with the convention that the points on the two surfaces with the same angular coordinates are identified. Since $H^{2}\left(S^{1} \times S^{2}\right)=\mathbb{Z}$, from relation (29) it follows that, as shown in Figure 2, $H_{D}^{1}\left(S^{1} \times S^{2}\right)$ can be understood as an affine bundle over $\mathbb{Z}$ in which each fibre has a linear space structure isomorphic to $\Omega^{1}\left(S^{1} \times S^{2}\right) / \Omega_{\mathbb{Z}}^{1}\left(S^{1} \times S^{2}\right)$.

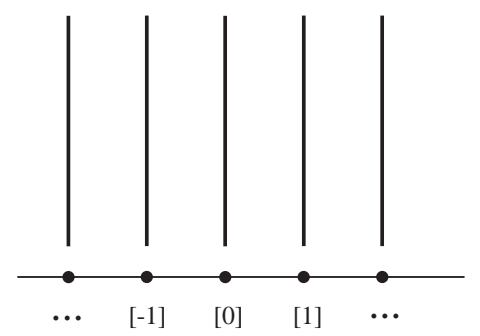

Figure 2: Presentation of the Deligne-Beilinson affine bundle $H_{D}^{1}\left(S^{1} \times S^{2}\right)$.

In order to fix an origin in $H_{D}^{1}\left(S^{1} \times S^{2}\right)$, we introduce the "diagonal" section $s$,

$$
\begin{aligned}
s: \mathbb{Z} & \rightarrow H_{D}^{1}\left(S^{1} \times S^{2}\right) \\
n & \mapsto s(n) \equiv n \gamma_{0},
\end{aligned}
$$

where $\gamma_{0} \in H_{D}^{1}\left(S^{1} \times S^{2}\right)$ denotes the DB class which is canonically associated with the knot $G_{0}$, shown in Figure 3 , that can be taken as a generator of $H_{1}\left(S^{1} \times S^{2}\right)=\mathbb{Z}$.

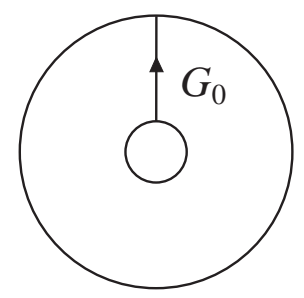

Figure 3: In the region of $\mathbb{R}^{3}$ that provides a description of $S^{1} \times S^{2}$, the oriented loop $G_{0} \subset S^{1} \times S^{2}$ - generator of $H_{1}\left(S^{1} \times S^{2}\right)$ - is represented.

Each element $A \in H_{D}^{1}\left(S^{1} \times S^{2}\right)$ can then be written as

$$
A=n \gamma_{0}+\alpha,
$$

for some integer $n$ and $\alpha \in \Omega^{1}\left(S^{1} \times S^{2}\right) / \Omega_{\mathbb{Z}}^{1}\left(S^{1} \times S^{2}\right)$. The (not regularized) functional 
measure takes the form

$$
\begin{aligned}
D A e^{2 \pi i k S_{C S}[A]} & =\sum_{n=-\infty}^{+\infty} D \alpha \exp \left\{2 \pi i k \int_{S^{1} \times S^{2}}\left(n \gamma_{0}+\alpha\right) *\left(n \gamma_{0}+\alpha\right)\right\} \\
& =\sum_{n=-\infty}^{+\infty} D \alpha \exp \left\{2 \pi i k \int_{S^{1} \times S^{2}} \alpha * \alpha\right\} \exp \left\{4 \pi i k n \int_{S^{1} \times S^{2}} \alpha * \gamma_{0}\right\} .
\end{aligned}
$$

Because of the non-trivial homology of the manifold $S^{1} \times S^{2}$, the functional measure has a physical (not related to the gauge invariance) zero mode $\beta_{0} \in H_{D}^{1}\left(S^{1} \times S^{2}\right)$. More precisely, let us represent a generator of $H_{2}\left(S^{1} \times S^{2}\right)$ by a oriented 2-dimensional sphere $S_{0}$; $S_{0}$ is isotopic with the component $S^{2}$ of $S^{1} \times S^{2}$ and, if one represents $S^{1} \times S^{2}$ by the region of $\mathbb{R}^{3}$ which is delimited by two concentric spheres, $S_{0}$ can just be represented by a third concentric sphere, as shown in Figure 4.

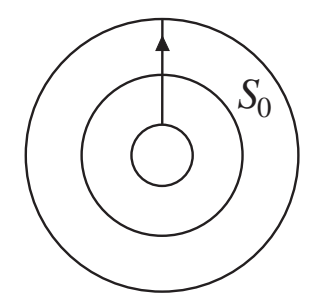

Figure 4: The sphere $S_{0} \subset S^{1} \times S^{2}$.

Let $\beta_{0}$ be the distributional 1-form which is globally defined in $S^{1} \times S^{2}$ and has support on $S_{0}$, and let $\beta_{0} \in H_{D}^{1}\left(S^{1} \times S^{2}\right)$ be the class which is locally described by the distributional 1-form $\beta_{0}$. The overall sign of $\beta_{0}$ is fixed by the orientation of $S_{0}$ so that

$$
\int_{G_{0}} \beta_{0}=1
$$

Since the boundary of the closed surface $S_{0}$ is trivial, one has $d \beta_{0}=0$. From equation (37) and $d \beta_{0}=0$ it follows that, for any integer $m$,

$$
\exp \left\{2 \pi i k S_{C S}[A]\right\}=\exp \left\{2 \pi i k S\left[A+(m / 2 k) \beta_{0}\right]\right\} \text {, }
$$

that implies

$$
\exp \left\{2 \pi i k S_{C S}[A]\right\}=\frac{1}{2 k} \sum_{m=0}^{2 k-1} \exp \left\{2 \pi i k S\left[A+(m / 2 k) \beta_{0}\right]\right\}
$$

Consider now the observable (28) associated with the link $L \subset S^{1} \times S^{2}$ for fixed integer $k$. The integral

$$
[L]=\int_{L} \beta_{0}
$$

takes integer values; the value of $[L]$ is equal to the sum of the intersection numbers (weighted with the values of the colour of the link components) of the link $L$ with the surface $S_{0}$. 
Property (39) implies that $\left.\langle W(L)\rangle\right|_{S^{1} \times S^{2}}$ vanishes unless $[L] \equiv 0 \bmod 2 k$ and, in that case, one can use again an identity of the type shown in equation (32) to get 15

$$
\left.\langle W(L)\rangle\right|_{S^{1} \times S^{2}}=\left\{\begin{array}{l}
0 \text { if }[L] \not \equiv 0 \bmod 2 k \\
\exp \left\{-(2 i \pi / 4 k) \sum_{i j} q_{i} \mathbb{L}_{i j} q_{j}\right\} \text { if }[L] \equiv 0 \bmod 2 k .
\end{array}\right.
$$

Expression (41) is the result of a real (non perturbative) functional integration computation in a non-trivial manifold. Equation (41) is in agreement with the prediction of the Reshetikhin-Turaev surgery rules, that will be discussed in the next section.

The model considered in this section is an example of topological field theory in which standard perturbation theory cannot be used. In the quantum Chern-Simons field theory formulated in $S^{1} \times S^{2}$, the standard Feynman propagator for the $A$ field does not exist because of the normalizable zero mode that corresponds to the class $\beta_{0} \in H_{D}^{1}\left(S^{1} \times S^{2}\right)$. Among the field configurations, one can find a globally defined 1-form $A_{0}$ such that $d A_{0}=0$ but $A_{0}$ is not the gauge transformed of something else.

The Deligne-Beilinson formalism has been also applied [15] to the torsion-free manifolds $M=S^{1} \times \Sigma_{g}$ where $\Sigma_{g}$ is a closed 2-surface of genus $g \geq 1$; and one example of manifold with torsion, $M=R P^{3}$, has been studied by Thuillier in [25].

\section{$5 \quad$ Surgery invariants}

In order to compute - by means of the quantum groups modular algebra - topological invariants in a generic 3-manifold $M$, Reshetikhin and Turaev have produced appropriate surgery rules [26] that are in agreement with the suggestions contained in 5]. These rules have been developed also by Kohno [27, by Lickorish [28] and by Morton and Strickland [29.

According to the Reshetikhin-Turaev surgery rules (adapted to the case of the abelian Chern-Simons field theory), for fixed integer $k$, the expectation value of the Wilson line $W(L)$ associated with a link $L$ in the 3 -manifold $M=M_{\mathcal{L}}$ can be written as a ratio

$$
\text { surgery rules: }\left.\quad\langle W(L)\rangle\right|_{M_{\mathcal{L}}}=\left.\langle W(L) W(\mathcal{L})\rangle\right|_{S^{3}} /\left.\langle W(\mathcal{L})\rangle\right|_{S^{3}} .
$$

In equation $(42), \mathcal{L}$ is the surgery link that codifies a Dehn surgery 30 presentation of $M$ in $S^{3}$; the integer surgery coefficients are determined by the framings of the components of $\mathcal{L}$. In the computation of the expectation values in $S^{3}$, one has to sum over the values $q=0,1,2, \ldots, 2 k-1$ of the colours which are associated with the components of $\mathcal{L}$. The structure of equation (42) is somehow similar to the structure of the corresponding functional integral expression

$$
\text { functional integral: }\left.\quad\langle W(L)\rangle\right|_{M}=\frac{\int_{M} D A e^{2 \pi i k S_{C S}[A]} W(L)}{\int_{M} D A e^{2 \pi i k S_{C S}[A]}} .
$$

But equation (42) is not based on a path-integral in the manifold $M$; the numerator and the denominator (which appear on the r.h.s of the equation) - that must be computed in 
$S^{3}$ - are separately well defined and, when the denominator is not vanishing, the ratio (42) is well defined. Expression (42) refers to a particular surgery presentation described by the surgery link $\mathcal{L}$ but, since the ratio (42) is invariant under Kirby moves [31, expression (42) is an isotopy invariant of the link $L$ in the oriented 3-manifold $M$.

In all the examples considered so far, the computations of the expectation values $\left.\langle W(L)\rangle\right|_{M}$ of equation (43), which have been obtained by means of the nonperturbative path-integral formalism based on the Deligne-Beilinson cohomology, are in agreement with expression (42).

Assuming that equations (42) and (43) are equivalent, one finds that for certain manifolds and for particular integer values of the coupling constant $k$, the path-integral formula (43) is not well defined. For instance, in the case $M=R P^{3}$ the denominator of the ratio (42) is vanishing when $k$ is an odd integer and then expression (42) is not well defined. In the functional integral approach, the constraint on the values of $k$ when $M=R P^{3}$ has been discussed in [25].

Equation (42) implies that [32] :

- the set of expectation values of the $U(1)$ Chern-Simons theory in $S^{3}$ and in any homology sphere $M_{0}$ coincide

$$
\left\{\left.\langle W(L)\rangle\right|_{M_{0}}\right\}=\left\{\left.\langle W(L)\rangle\right|_{S^{3}}\right\} .
$$

- For any coloured, oriented and framed link $L \subset M$, one can introduce a new link $\widetilde{L} \subset M$ (called the simplicial satellite of $L$ ) which is a satellite of $L$ and which is obtained from $L$ by replacing each link component of colour $q$ with $|q|$ parallel copies of the same component, each copy with unitary colour. If the simplicial satellite $\widetilde{L}$ of the link $L$ in a generic manifold $M$ is homologically trivial $\bmod 2 k$, then there exists a link $L^{\prime}$ in $S^{3}$ such that

$$
\left.\langle W(L)\rangle\right|_{M}=\left.\left\langle W\left(L^{\prime}\right)\right\rangle\right|_{S^{3}} .
$$

By means of the Reshetikhin-Turaev surgery rules, for fixed integer $k$, one can also define a 3-manifold invariant

$$
I_{k}(M)=I_{k}\left(M_{\mathcal{L}}\right)=\left.(2 k)^{-N_{\mathcal{L}} / 2} e^{i \pi \sigma(\mathcal{L}) / 4}\langle W(\mathcal{L})\rangle\right|_{S^{3}},
$$

where $N_{\mathcal{L}}$ denotes the number of components of $\mathcal{L}$ and $\sigma(\mathcal{L})$ represents the so-called signature of the linking matrix associated with $\mathcal{L}$, i.e. $\sigma(\mathcal{L})=n_{+}-n_{-}$where $n_{ \pm}$is the number of positive/negative eigenvalues of the linking matrix which is defined by the framed link $\mathcal{L}$. Some properties of $I_{k}\left(M_{\mathcal{L}}\right)$ (and of its generalizations) have been studied, for instance, in [33, 34, 35. If $M_{0}$ is a homology 3-sphere, then [32] one finds $I_{k}\left(M_{0}\right)=1$. One could suspect that the invariant $I_{k}(M)$ only depends on the homology group $H_{1}(M)$; the following counterexamples show that this is not the case. The lens spaces $L(5,1)$ and $L(5,2)$ are not homeomorphic but they have the same homology group $\mathbb{Z}_{5}$; equation (46) gives

$$
I_{2}(L(5,1))=-1 \quad, \quad I_{2}(L(5,2))=1 .
$$


Similarly, the manifolds $L(9,1)$ and $L(9,2)$ are not homeomorphic; they have the same homology group $\mathbb{Z}_{9}$ and are of the same homotopy type. One finds

$$
I_{3}(L(9,1))=i \sqrt{3} \quad, \quad I_{3}(L(9,2))=-i \sqrt{3} .
$$

\section{Partition function}

In order to complete the answer to the question formulated in the Introduction, let us recall that, in quantum field theory, any well defined functional integration takes really the form of a ratio of functional integrations. Therefore one can imagine that the "suitably normalized" partition function of the Chern-Simons theory formulated in the closed 3-manifold $M$,

$$
R_{N_{0}}(M)=\frac{\int_{M} D A e^{i S_{C S}}}{N_{0}},
$$

should correspond to the Reshetikhin-Turaev surgery invariant for the manifold $M$. Equation (49) should be interpreted as the result of some limit prescription for the ratio of two regularized functional integrals, as indicated in equation (6). So, $N_{0}$ stands for an appropriate path-integral that introduces a reference point for the integration. Presumably, $N_{0}$ is not unique; the specific choice of $N_{0}$ is precisely the crucial point that will make expression (49) well defined.

Several variants of the Reshetikhin-Turaev surgery invariant have been introduced in literature and have been used to obtain well defined results; but all these combinatorial invariants are not really based on a functional integration. We hope that, in the future, a true functional integral derivation of an explicit and well defined expression of a 3-manifold invariant will be produced.

Acknowledgments. I wish to thank F. Thuillier for useful discussions.

\section{References}

[1] V.W. Hughes and T. Kinoshita, Rev. of Mod. Phys. 71 (1999) S133; B. Odom, D. Hanneke, B. D'Urso and G. Gabrielse, Phys. Rev. Lett. 97, 030801 (2006); G. Gabrielse, D. Hanneke, T. Kinoscita, M. Nio and B. Odom, Phys. Rev. Lett. 97, 030802 (2006).

[2] N.N. Bogolioubov et D.V. Chirkov, Introduction a la théorie quantique des champs, Dunod Editeur (Paris, 1960).

[3] C. Itzykson and J-B. Zuber, Quantum Field Theory, McGraw-Hill (Singapore, 1980).

[4] J. Schwinger, Phys. Rev. 82 (1951) 664.

[5] E. Witten, Commun. Math. Phys. 121 (1989) 351.

[6] F. Delduc, O. Piguet, C. Lucchesi and S.P. Sorella, Nucl. Phys. B 346 (1990) 313. 
[7] A.C. Hirshfeld and U. Sassenberg, Journal of Knot Theory and its Ramifications, 5 (1996) 805.

[8] V.F.R. Jones, Ann. Math. 126 (1987) 335.

[9] P. Freyd, D. Yetter, J. Hoste, W.B.R. Lickorish, K. Millett and A. Ocneanu, Bull. Amer. Math. Soc. 12 (1985) 239; J.H. Przytycki and P. Traczyk, Kobe J. Math. 4 (1987) 115.

[10] V.G. Turaev, Invent. Math. 92 (1988) 527.

[11] L.H. Kauffman, Topology 26 (1987) 395.

[12] V.G. Knizhnik and A.B. Zamolodchikov, Nucl. Phys. B 247 (1984) 83.

[13] T. Kohno, Ann. Inst. Fourier, Grenoble, 37 (1987) 139.

[14] A. Tsuchiya and Y. Canie, Adv. Stud. Pure Math. 16 (1988) 297.

[15] E. Guadagnini and F. Thuillier, SIGMA 4 (2008) 078, 30 pages, arXiv:0801.1445.

[16] A.S. Schwarz, Lett. Math. Phys. 2 (1978) 247.

[17] A.S. Schwarz, Commun. Math. Phys. 67 (1979) 1.

[18] C.R. Hagen, Ann. Phys. 157 (1984) 342.

[19] M. Bauer, G. Girardi, R. Stora and F. Thuillier, JHEP 0508 (2005) 027.

[20] P. Deligne, Théorie de Hodge II, Publ. Math. I.H.E.S., 40 (1971) 5.

[21] A.A. Beilinson, J. Soviet Math. 30 (1985) 2036.

[22] J. Cheeger and J. Simons, Differential characters and geometric invariants, Stony Brook Preprint 1973; reprinted in Lecture Notes in Mathematics 1167, Geometry and Topology Proc. 1983-84, Eds J. Alexander and J. Harer, Springer 1985.

[23] J.L. Brylinski, Loop spaces, characteristic classes and geometric quantization, Progress in Mathematics 107 (Boston, 1993).

[24] R. Harvey, B. Lawson and J. Zweck, Amer. J. Math. 125 (2003) 791.

[25] F. Thuillier, J. Math. Phys. 50, 122301 (2009); arXiv:0901.2485.

[26] N.Y. Reshetikhin and and V.G. Turaev, Commun. Math. Phys. 127 (1990) 1; and Invent. Math. 103 (1991) 547.

[27] T. Kohno, Topology 31, (1992), 203.

[28] W.B.R. Lickorish, Pacific J. Math. 149 (1991) 337. 
[29] H.R. Morton and P.M. Strickland, Satellites and Surgery Invariants, in Knots 90, ed. A. Kawauchi, Walter de Gruyter, Berlin, 1992.

[30] D. Rolfsen, Knots and Links, AMS Chelsea Publishing, Providence, Rhode Island, 2003.

[31] R. Kirby, Invent. Math. 45 (1978) 35.

[32] E. Guadagnini and F. Mancarella, work in progress.

[33] H. Murakami, T. Ohtsuki and M. Okada, J. Math. 29 (1992) 545.

[34] F. Deloup, C. R. Acad. Sci. Paris, t. 326, Série i, (1998) 69.

[35] S.K. Hansen, J.K. Slingerland and P.R. Turner, Adv. Theor. Math. Phys. 9 (2005) 321. 